\title{
Les freins juridiques à la transition agro-écologique
}

Antoine de Lombardon, Avocat à la Cour

Benoît Grimonprez, Professeur d'Université - Institut de droit rural de Poitiers

En dépit d'un engouement sociétal pour une agriculture plus respectueuse des hommes et de la nature, des freins juridiques persistent et entravent l'évolution des pratiques vers l'agroécologie. Cette dernière notion a pourtant été promue par la loi $n^{\circ}$ 2014-1170 du 13 octobre 2014 comme la combinaison d'une triple performance, économique, sociale et environnementale, des exploitations agricoles. Les objectifs politiques de la transition ont beau désormais figurer dans les textes, les mesures techniques qui permettraient la libération de nouvelles formes d'agriculture font toujours défaut. Quatre illustrations emblématiques peuvent être données de cette résistance normative qui, avec de la volonté, pourrait être vaincue.

\section{L'inadaptation du statut du fermage}

Le bail rural soumis au statut du fermage comporte, tout d'abord, d'importantes lacunes au plan environnemental. La raison tient au principe selon lequel le fermier jouit d'une liberté, presque totale, d'exploiter le fonds.

Au sein d'un bail rural ordinaire, il n'existe pas vraiment de prise en compte des qualités écologiques des biens loués. Ainsi la mauvaise gestion environnementale du fonds est-elle rarement sanctionnée. Sauf à supprimer, sans autorisation, certains éléments naturels (arbres, haies ${ }^{1}$ ) ou à polluer les lieux, le preneur n'encourt pas de résiliation du bail, ni de paiement d'une indemnité pour dégradation du bien lorsqu'il use de méthodes de culture peu écologiques. Par ailleurs, il est interdit au bailleur d'orienter la production de son fermier vers des circuits de distribution plus courts ou locaux. Cela vaut y compris pour les bailleurs personnes publiques qui louent des terres leur appartenant : ils ne peuvent mettre l'agriculture qu'ils soutiennent au service de leurs administrés.

Certes le bail rural peut maintenant inclure des clauses environnementales. Mais cette possibilité reste exceptionnelle et ne peut intervenir que dans des cas limitativement énumérés (en fonction des zones, du type de bailleur, ou des pratiques et infrastructures préexistantes) ${ }^{2}$. II est pour le moins incongru, à notre époque, que la liberté de stipuler des clauses environnementales dans les baux ne soit pas conquise.

Le statut du fermage s'acclimate, ensuite, difficilement à l'agriculture urbaine. Le phénomène est pourtant en plein essor. Le 15 octobre 2015, sept métropoles françaises ont signé le pacte de Milan relatif à la politique alimentaire urbaine ${ }^{3}$. Ce texte invite les villes signataires à

\footnotetext{
${ }^{1}$ Pour un exemple de résiliation de bail et condamnation à dommage-intérêts en raison de l'arrachage de haies : CA Versailles 27 mai 2014, confirmé par C. Cass. $3^{\text {ème }}$ Civ. $1^{\text {er }}$ oct. 2014, n¹3-22306.

2 C. rur., art. L. 411-27.

${ }^{3}$ Marseille, Bordeaux, Grenoble, Lyon, Montpellier, Nantes et Paris ont signé ce document.
} 
développer une politique alimentaire sur leur territoire, laquelle passera par des initiatives en tous genres, en zones périurbaines ou urbaines, par de nouveaux acteurs, sur de nouveaux supports, et au moyen de nouvelles techniques.

Les agriculteurs urbains se heurtent cependant au statut intangible des baux ruraux qui a été pensé et conçu en regard des fonds de terre. L'application du droit des champs à l'agriculture des villes pose plusieurs difficultés.

Il faut savoir que le régime d'ordre public des baux ruraux s'applique dès lors qu'un immeuble est loué en vue de l'exercice d'une activité agricole (C. rur., art. L. 411-1). Cela entraîne, en l'occurrence, des conséquences fâcheuses. La fixation du loyer est obligatoirement comprise entre des minima et maxima (peu élevés en pratique), tandis que le bail est conclu pour une durée minimale de 9 ans, avec un droit au renouvellement pour le preneur. Surtout, le locataire peut se voir interdire, par une clause, de déployer des activités commerciales sur les lieux loués, avec la résiliation du bail comme sanction. Or, cette pluriactivité apparaît très présente dans la plupart des projets agri-urbains. Autre inconvénient de taille, le Code rural permet au propriétaire dont les biens sont situés en zone urbaine d'un plan local d'urbanisme de résilier le bail à tout moment, par une simple notification (C. rur., art. L. 411-32). L'instabilité juridique de la relation locative est donc forte pour l'exploitant.

Pour échapper au statut du fermage, nombre d'agriculteurs urbains sont incités à souscrire une convention d'occupation précaire, sur la base de l'article L. 411-2 du Code rural qui l'autorise, en particulier, sur les biens " dont l'utilisation principale n'est pas agricole ou dont la destination agricole doit être changée ». Dans cet esprit, on considère donc que le toit, la cave et la friche urbaine accueillant l'activité agricole n'auraient pas une vocation agricole principale ou pérenne. Or cette lecture peut être contestable à l'heure où les villes ouvrent durablement des espaces à la production alimentaire ${ }^{4}$. Aussi est-il probable que les conventions litigieuses fassent l'objet d'une requalification en bail soumis au statut du fermage. Les dispositions, plus souples, concernant les baux de petites parcelles ne pourront que rarement bénéficier aux agriculteurs urbains (C. rur., art. L. 411-3). En effet, ces règles ne sauraient s'appliquer pour les biens loués constituant «les parties essentielles d'une exploitation agricole ».

Au regard de ces réflexions, on comprend les initiatives ${ }^{5}$ plaidant pour une adaptation - sinon une dérogation - du statut du fermage aux particularités de l'agriculture urbaine. II manque à l'évidence une figure contractuelle, stabilisant les agriculteurs urbains et offrant aux propriétaires des perspectives de valorisation de l'immeuble conformes à son environnement.

\footnotetext{
${ }^{4} \mathrm{~A}$ titre d'exemple, la Ville de Paris a cherché à mettre plus de 50 hectares à disposition de projets d'agriculture urbaine.

${ }^{5}$ Proposition de loi ${ }^{\circ} 786$ du 21 mars 2018 «visant à revitaliser les terres agricoles en zone périurbaine » en vue de permettre aux SCOT de délimiter des aires urbaines de production agricole au sein desquelles pourront être souscrits des baux dérogatoires au statut du fermage (durée de 4 ans, interdiction de construction de bâtiments, absence d'indemnité de fin de bail...).
} 


\section{La dilution de l'agro-écologie au sein du contrôle des structures}

L'agro-écologie fait théoriquement partie intégrante du contrôle des structures, régime administratif par lequel le préfet de Région autorise les opérations d'installation, d'agrandissement et de réunion d'exploitations (C. rur., art. L. 331-2). Les considérations agroécologiques apparaissent bien au sein des objectifs du contrôle, mais sont désespérément absentes au stade de son déclenchement, ainsi que de l'octroi de l'autorisation.

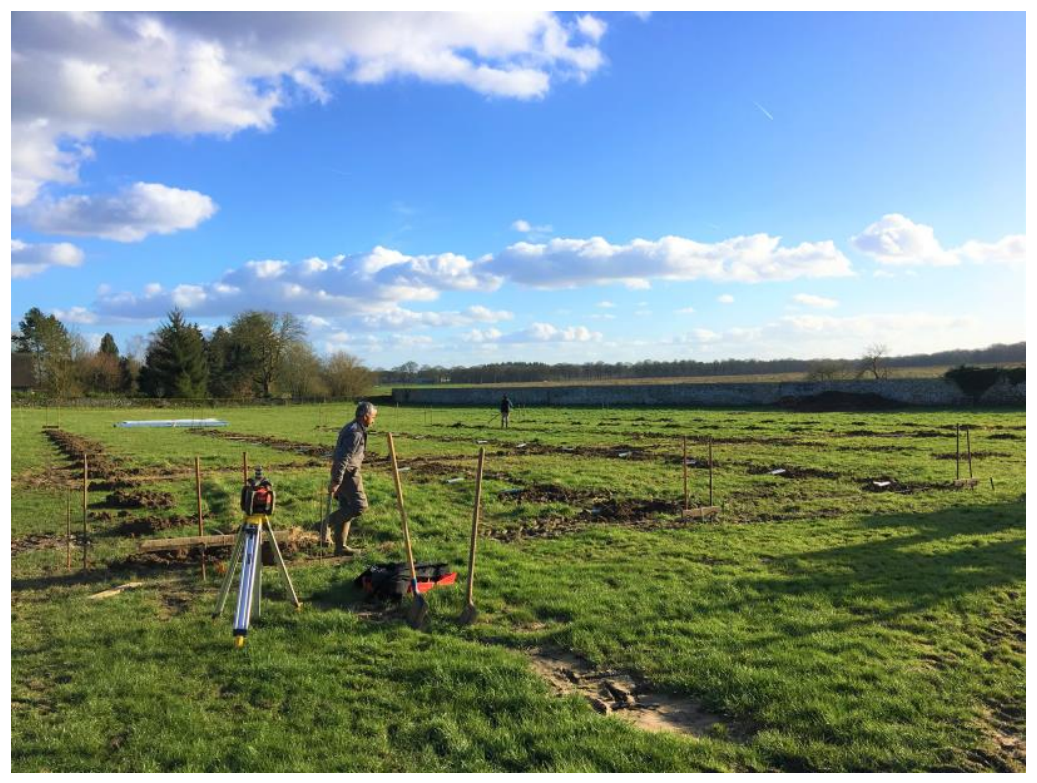

La promotion de systèmes agro-écologiques compte parmi les objectifs du contrôle (C. rur., art. L. 331-1, $2^{\circ}$ ). Elle n'est cependant pas prioritaire, comparée à l'installation d'agriculteurs, laquelle est favorisée sans égard pour le mode de culture envisagé par le nouvel exploitant.

Les critères de soumission au contrôle des structures ignorent également la dimension environnementale de l'activité projetée. Seuls importent, pour déterminer le régime d'autorisation ou de déclaration d'exploiter, les surfaces mises en valeur ainsi que la personnalité des exploitants, notamment à travers leur capacité professionnelle ou leur appartenance au cercle familial (C. rur., art. L. 331-2).

Au niveau de l'octroi de l'autorisation, il faut s'en remettre aux ordres de priorité des différents Schémas Directeurs Régionaux des Exploitations Agricoles (SDREA) pour voir si la mise en œuvre de méthodes agro-écologiques permet à l'exploitant, en concurrence avec d'autres, d'obtenir un meilleur classement. Pour rappel, les contenus des SDREA sont le fruit d'arbitrages intervenus, pour l'essentiel, entre les différents représentants de la profession agricole $^{6}$. Or, cette dernière mésestime encore l'agro-écologie. Si certains schémas (par ex. en Bretagne) lui accordent une place parmi les ordres de priorité (encouragement du pâturage,

${ }^{6}$ Les syndicats agricoles représentent $25 \%$ des membres des CDOA (soit 8 membres sur 32, alors que les associations environnementales comptent 2 membres). 
promotion de l'agriculture biologique, systèmes plus économes en intrants...), ils promeuvent aussi les critères liés à l'installation, à la taille de l'exploitation, au nombre d'emplois maintenus... Le résultat est une dilution des considérations agro-écologiques qui conduit, la plupart du temps, à privilégier des projets agricoles plus conventionnels et moins innovants.

Plusieurs pistes sont envisageables pour remédier à la situation. On pourrait d'abord dispenser du régime d'autorisation d'exploiter les agriculteurs qui déploient des méthodes agroécologiques dans la mesure où sont en jeu des surfaces raisonnables. Pour ces candidats au foncier, une simple déclaration pourrait parfaitement suffire. Une autre solution serait ensuite de faire de l'engagement agro-écologique un critère prioritaire des SDREA, de nature à conférer à l'exploitant un "super privilège », par exemple dans le cadre d'une installation. L'intérêt évident serait d'orienter le renouvellement des générations vers une forme d'agriculture plus durable et souvent plus rentable. II est enfin imaginable de subordonner l'octroi de l'autorisation administrative d'exploiter au respect d'un cahier des charges, en particulier environnemental (gestion des sols, de la biodiversité, de l'eau...). Contrairement à ce qui existe aujourd'hui, l'administration assurerait alors un suivi dans le temps des conditions d'exploitation?.

\section{L'intégration laborieuse de l'environnement au sein des signes d'identification de la qualité et de l'origine}

Les Signes d'Identification de la Qualité et de l'Origine (SIQO) permettent de différencier et de valoriser les produits agricoles. Ils englobent les Appellations d'Origine Protégée, les Indications Géographiques Protégées, les Spécialités Traditionnelles Garanties, l'Agriculture Biologique et le Label Rouge. A l'exception notable de l'Agriculture Biologique, les autres signes n'intègrent pas nécessairement d'exigences environnementales à leurs cahiers des charges.

La prise en compte de la donnée agronomique par les SIQO est une question ancienne, déjà apparue dans le domaine viticole. A l'origine, les lois du $1^{\text {er }}$ août 1905 et 5 août 1908 sur la protection de l'origine du vin s'étaient focalisées sur la provenance des produits et l'identification des périmètres de production. Les méthodes de culture et les conditions de production étaient ignorées. II a fallu attendre le décret-loi du 30 juillet 1935, adopté sous l'influence du député Joseph Capus, pour que ces éléments fassent partie des cahiers des charges des appellations d'origine contrôlées ${ }^{8}$. II n'en reste pas moins que les critères proprement environnementaux sont aujourd'hui sommaires dans la plupart des appellations ou signes de qualité (ex. enherbement partiels des vignes, proscription des OGM...). Une plus grande ambition est attendue pour des produits censés représenter l'excellence du patrimoine gastronomique français.

\footnotetext{
7 Sur ces propositions: B. Grimonprez, L'arable du futur: RD rur. 2018, Dossier 15 : https://hal.archivesouvertes.fr/hal-01838706

${ }^{8} \mathrm{~N}$. Olszak, Le vin de terroir et la naissance des appellations d'origine, in Le droit du vin à l'épreuve des enjeux environnementaux, ed. Féret, 2015, p. 51.
} 
La loi dite «Egalim » du 30 octobre 2018 (art. 48) est récemment intervenue pour imposer, par voie de décret, aux SIQO d'intégrer à leurs cahiers des charges, d'ici le $1^{\text {er }}$ janvier 2030 , la nécessité pour les opérateurs de répondre aux exigences de la certification environnementale.

Selon l'article L. 611-6 du Code rural, la certification environnementale permet de reconnaître la performance environnementale des exploitations en termes de biodiversité, de stratégie phytosanitaire, de gestion des fertilisants et de ressource en eau. Elle comprend trois niveaux, le troisième étant le plus élevé avec la labellisation « Haute Valeur Environnementale ». Le niveau 2, beaucoup moins contraignant, correspond à l'ancienne référence à l'agriculture raisonnée. Quant au niveau 1, il impose de simples obligations documentaires : (i) un bilan montrant que l'exploitant est en règle au regard des normes environnementales applicables à son exploitation (respect des prescriptions réglementaires en matière de gestion et de bonnes conditions agricoles et environnementales) et (ii) une évaluation de la situation de l'exploitation par rapport aux normes de référence des niveaux 2 et 3 de la certification.

La prescription formulée par la loi du 30 octobre 2018 demeure cependant assez floue quant à sa portée. Littéralement, une certification environnementale mentionnée à l'article L. 611-6 sera exigée, sans précision de son niveau ${ }^{9}$. Or le niveau 1 permet le maintien d'un mode de culture sans performance environnementale particulière. Le texte pourrait donc se montrer particulièrement décevant, d'autant que l'échéance fixée pour atteindre cet objectif minimal est très éloignée. Tout dépendra, à vrai dire, du décret d'application de cette disposition. Reste à souhaiter que la plupart des cahiers de charges fasse sa révolution, avec des comités de défense et de gestion bien plus audacieux.

Tandis que les signes de qualité peinent à se saisir de la transition agro-écologique, d'autres pratiques commerciales apparaissent, qui brouillent tous les repères. C'est le cas des allégations de nature environnementale qui pullulent sur de nombreux produits sous l'égide de marques privées («produit bon et sain », « respectueux de la nature », « démarche agroécologique »...). Or, l'encadrement réglementaire et le contrôle de ces types de mentions valorisantes est quasiment inexistant. S'ensuit que beaucoup d'affirmations, à des fins purement publicitaires, sont délibérément ambigües et vagues, donc souvent trompeuses pour le consommateur ${ }^{10}$. Le succès de ce genre de méthodes commerciales contribue, en fin de compte, au maintien des vieilles pratiques agricoles dont seule la méthode de promotion a changé.

\section{La restriction à la mise sur le marché des semences paysannes}

\footnotetext{
${ }^{9}$ Sur ces discussions parlementaires, v. Assemblée Nationale, 1 ère lecture, $3^{\text {ème }}$ séance du 26 mai $2018, M$. Dominique Potier à propos de l'amendement $n^{\circ} 2466$ : «notre amendement propose une démarche très progressive, avec (...) un respect des règles de la certification HVE de niveau 1, 2 ou 3 pour tous les cahiers de charges d'ici à 2030 ». V. aussi, Assemblée Nationale, 1ère lecture, 3ème séance du 26 mai 2018, Monsieur JeanBaptiste Moreau : «j'émets un avis favorable à l'amendement 2466 rectifié qui indique que les SIQO reçoivent, d'ici à 2030, une certification de niveau HVE 1 au moins ».

${ }^{10}$ Lequel paie généralement plus cher cette gamme de produits, sans avoir pour autant l'assurance d'une plusvalue qualitative.
} 
Voilà 30 ans que la FAO a alerté sur la raréfaction des ressources phytogénétiques. Depuis $1900,75 \%$ de la biodiversité agricole a disparu à l'échelle mondiale. La sélection industrielle des semences, protégée par des droits de propriété intellectuelle (brevets, certificats d'obtention végétale), est l'une des causes de cet effondrement. Un tel système peut s'accompagner, comme en France, de la pénalisation de la mise sur le marché de variétés de semences qui ne sont pas inscrites au catalogue officiel des variétés ${ }^{11}$.

Pour figurer sur ce catalogue, une variété doit répondre à trois critères : être distincte d'une autre variété (distinction), être composée de plantes homogènes entre elles (homogénéité), et être stable au cours des reproductions successives (stabilité). Un tel régime se justifie par un but exclusivement économique. Hélas, le critère d'homogénéité tend à rétrécir la base génétique des plantes dans une logique de standardisation des individus. Quant au critère de la distinction, il porte souvent sur des caractères phénotypiques tellement précis qu'il conduit, en pratique, à inscrire de nouvelles variétés végétales assez peu différentes des anciennes. D'où la mise sur le marché de semences de plus en plus apparentées entre elles. En France, une étude de la Fondation pour la Recherche sur la Biodiversité ${ }^{12}$ a ainsi démontré, pour le cas du blé tendre, qu'entre 1912 et 2006 la biodiversité génétique de ces variétés avait globalement diminué.

En l'état actuel du droit français, vendre une variété végétale qui n'est pas inscrite au catalogue est passible d'une contravention de $5^{\text {ème }}$ classe, soit jusqu'à 3000 euros d'amende ${ }^{13}$.

La prohibition n'est pas absolue car elle ne porte que sur la mise sur le marché d'une variété en vue de son « exploitation commerciale» (D. $n^{\circ}$ 81-605, 18 mai 1981, art. $1^{\mathrm{er}}$ ). Cela devrait, en principe, faire échapper à toute condamnation la vente de semences à l'attention des jardiniers amateurs qui ne revendent pas les fruits ou légumes issus de leur culture.

Telle n'était pourtant pas la position de la jurisprudence, la Cour d'appel de Nancy ayant par exemple considéré, dans un arrêt du 9 septembre $2014^{14}$, que la vente à des jardiniers amateurs constitue l'exploitation commerciale de la variété. Pour corriger cette interprétation, le législateur est intervenu, par deux fois, pour légaliser la distribution de variétés hors catalogue aux jardiniers amateurs. D’abord, la loi n²016-1087 du 8 août 2016 (art. 11) a consacré la liberté de céder et d'échanger gratuitement, au profit d'utilisateurs non professionnels, des matériels de reproduction des végétaux dont les variétés font partie du domaine public. Le Conseil constitutionnel a cependant censuré la partie du texte qui créait, pour les associations, une dérogation leur permettant de vendre (et non plus seulement de

\footnotetext{
${ }^{11}$ L'article L. 661-8 du Code rural et de la pêche maritime et le décret n`81-605 du 18 mai 1981 imposent cette inscription.

${ }^{12}$ R. Goffaux, I. Goldringer, C. Bonneuil, P. Montalent et I. Bonnin, "Quels indicateurs pour suivre la diversité génétique des plantes cultivées ? Le cas du blé tendre en France depuis un siècle ", Série Ressources Génétiques, FRB 2011.

${ }^{13}$ C. conso., art. R. 451-1.

${ }^{14}$ CA Nancy, 9 sept. 2014, n¹785/2014.
} 
donner) ce type de semences ${ }^{15}$. S'ensuit que la mesure n'autorise toujours pas la commercialisation des semences «paysannes » non enregistrées au Catalogue. Ensuite, la loi Egalim n²018-938 du 30 octobre 2018 (art. 78) a voulu permettre la cession «à titre onéreux » de telles semences, toujours à destination d'utilisateurs non professionnels. Mais la disposition n'a pas passé le cap du Conseil constitutionnel, qui lui a reproché une adoption non conforme à la procédure législative ${ }^{16}$.

Le développement des variétés populations ${ }^{17}$ se heurte donc à des obstacles certains. On devine pourtant qu'il s'agit de l'une des clés de l'adaptation des cultures au changement climatique. Une solution a minima consisterait à lever la sanction pénale liée à leur commercialisation. Sur le fond sinon, il faudrait revoir les conditions d'inscription au catalogue officiel, ainsi que reconnaître aux cultivateurs un privilège pour la sélection des semences qu'ils opèrent in situ ${ }^{18}$.

A travers ces morceaux de droit choisis, on voit que le chemin reste encore long pour adapter notre système juridique agricole aux nouvelles préoccupations environnementales. Derrière la façade d'objectifs verdoyants, de nombreuses règles demeurent empreintes d'une logique productiviste qui ne correspond plus aux besoins actuels, tant de la société que des agriculteurs eux-mêmes.

\footnotetext{
15 Cons. Const. n²016-737, DC 4 août 2016.

16 Cons. Const. n²018-771, DC 25 oct. 2018.

17 «Variété hétérogène formée de mélanges d'individus sélectionnés principalement par les agriculteurs dans leurs champs » : http://www.inra.fr/Chercheurs-etudiants/Systemes-agricoles/Tous-les-dossiers/Selection-classique-ouparticipative-plusieurs-strategies-pour-les-bles-bios/Varietes-populations-privilegier-l-adaptabilite/(key)/1.

18 B. Grimonprez, «Semences agricoles : la tragédie d'un bien commun », RD rur. 2017, Etude 31.
} 